\title{
Stefan-Boltzmann Law and Casimir Effect for the Scalar Field in Phase Space at Finite Temperature
}

\author{
R. G. G. Amorim $\mathbb{D}^{1},{ }^{1}$ J. S. da Cruz Filho, ${ }^{2}$ A. F. Santos $\mathbb{D},{ }^{2}$ and S. C. Ulhoa $\mathbb{D}^{3}$ \\ ${ }^{1}$ Faculdade Gama, Universidade de Brasília, Setor Leste (Gama), 72444-240 Brasília, DF, Brazil \\ ${ }^{2}$ Instituto de Física, Universidade Federal de Mato Grosso, 78060-900, Cuiabá, Mato Grosso, Brazil \\ ${ }^{3}$ Instituto de Física, Universidade de Brasília, 70910-900 Brasília, DF, Brazil \\ Correspondence should be addressed to S. C. Ulhoa; sc.ulhoa@gmail.com
}

Received 23 February 2018; Revised 24 May 2018; Accepted 20 June 2018; Published 7 August 2018

Academic Editor: Luca Stanco

Copyright (C) 2018 R. G. G. Amorim et al. This is an open access article distributed under the Creative Commons Attribution License, which permits unrestricted use, distribution, and reproduction in any medium, provided the original work is properly cited. The publication of this article was funded by $\mathrm{SCOAP}^{3}$.

\begin{abstract}
We use the scalar field constructed in phase space to analyze the analogous Stefan-Boltzmann law and Casimir effect, both of them at finite temperature. The temperature is introduced by Thermo Field Dynamics (TFD) formalism and the quantities are analyzed once projected in the space of coordinates. We show that using the framework of phase space it is possible to introduce a thermal energy which is related to temperature as it vanishes when the temperature tends to zero. In fact given such a correlation the formalism of TFD is equivalent when project is in momenta space when compared to coordinates space.
\end{abstract}

\section{Introduction}

Eugene Paul Wigner $[1,2]$ introduced in 1932 the first formalism to quantum mechanics in phase space, motivated by finding a way to treat transport equations for superfluids. Wigner formalism allows mapping between quantum operators, say A, defined in the Hilbert space, $\delta$, with classical functions, say $a_{w}(q, p)$, in phase space $\Gamma$, through the $*$ being the starproduct or Moyal-product. The opposite problem, i.e., from a classical function finding the corresponding operator, is accomplished by Weyl mapping. The main motivation to define a given physical theory in phase space is its natural interpretation as demonstrated by classical mechanics. It is not a simple task; for instance, the uncertainty principle is problematic to be formulated. In addition in Wigner's approach there is no true probability distribution. On the other hand a general framework such as the phase space allows one to analyze a certain system under a different perspective.

The star-product has been explored in phase space in different ways. Particularly, it has been used to define operators like $a_{w}(q, p) *$ of interest to study irreducible unitary representations of kinematical groups in phase space [3]. In case of nonrelativistic symmetries, this leads to a Schrödinger equation in phase space, where the wave function is directly associated with Wigner function, so with full physical meaning. In this formalism of quantum mechanics, the observables are represented by operators of type $\widehat{a}=a_{w} \star$, which are used to construct a representation of Galilei symmetries. The Wigner function is given by $f_{w}(q, p)=\psi \star \psi^{\dagger}$ where $\psi=\psi(q, p)$ are the wave functions, solutions of Schrödinger equation represented in phase space. Since it is a theory of representation, this formalism has been generalized to the relativistic case, leading to the Klein-Gordon and Dirac equations in phase space [4]. This method has been applied successfully, for instance, to the analysis of abelian gauge symmetries [5], describing the dynamics (interaction) in the formulation of quantum theory in phase space.

Although the quantum field theory has successfully been applied to several systems, it does not take into account the temperature of such systems. This is a fundamental problem since all macroscopic features of a quantum model are related to temperature. Among all schemes to introduce temperature in a physical theory we cite two ways. The first one is to interpret time as temperature by a Wick rotation [6]. This approach is problematic when one is dealing with a time-evolution of a physical state. The second one the socalled Thermo Field Dynamics (TFD) formalism; it is a 
natural way to deal with dynamical systems. It preserves the time-evolution once the temperature is identified with a rotation in a duplicated Fock space [7]. In this article we explore how to implement TFD in phase space; particularly we analyze the scalar field in phase space at finite temperature. The Casimir effect for scalar field in phase space at zero and finite temperature is calculated.

The Casimir effect [8] is measured when two parallel conducting plates are attracted due to vacuum fluctuations. Although the first application has been developed for the electromagnetic field all quantum fields should exhibit this phenomenon. In fact, it was demonstrated that in nonrelativistic fields such as in Schrödinger equation the Casimir effect is present [9]. Particularly for nonrelativistic fields such an effect is physical only at finite temperature. Sparnaay [10] made the first experimental observation of the Casimir effect. Subsequent experiments have established this effect to a high degree of accuracy $[11,12]$.

The article is divided as follows. In Section 2, the symplectic Klein-Gordon field is introduced. In Section 3, we show the canonical quantization of the scalar field. Then, in Section 4, we recall the ideas of Thermo Field Dynamics formalism. In Section 5, we calculate the energy-momentum tensor of the symplectic scalar field and in Section 6 we derive the Stefan-Boltzmann-like law and the Casimir effect at finite temperature. Finally, in the last section we present our conclusions.

\section{Symplectic Klein-Gordon Field and Wigner Function}

Let us define the star-operator as

$$
\begin{aligned}
\widehat{A} & =A(q, p) \star \\
& =A(q, p) \exp \left[\frac{i \hbar}{2}\left(\frac{\overleftarrow{\partial}}{\partial q} \frac{\vec{\partial}}{\partial p}-\frac{\overleftarrow{\partial}}{\partial p} \frac{\vec{\partial}}{\partial q}\right)\right] \\
& =A\left(q+\frac{i \hbar}{2} \partial_{p}, p-\frac{i \hbar}{2} \partial_{p}\right),
\end{aligned}
$$

and hence we can derive the following operators:

$$
\begin{aligned}
& \widehat{P}^{\mu}=p^{\mu} \star=p^{\mu}-\frac{i}{2} \frac{\partial}{\partial q_{\mu}}, \\
& \widehat{Q}^{\mu}=q^{\mu} \star=q^{\mu}+\frac{i}{2} \frac{\partial}{\partial p_{\mu}},
\end{aligned}
$$

and

$$
\widehat{M}_{\nu \sigma}=M_{\nu \sigma} \star=\widehat{Q}_{\nu} \widehat{P}_{\sigma}-\widehat{Q}_{\sigma} \widehat{P}_{\nu}
$$

These operators satisfy Poincaré algebra and act in Hilbert space associated with phase space $\mathscr{H}(\Gamma)$. From them, we construct a symplectic representation of Poincaré-Lie algebra and, as a result, we obtain the Klein-Gordon equation in phase space [4]:

$$
\begin{aligned}
& \widehat{P}^{2} \phi(q, p)=p^{2} \star \phi(q, p)=m^{2} \phi(q, p) \\
& \left(p^{\mu} p_{\mu}-i p^{\mu} \frac{\partial}{\partial q^{\mu}}-\frac{1}{4} \frac{\partial}{\partial q^{\mu}} \frac{\partial}{\partial q_{\mu}}\right) \phi(q, p)=m^{2} \phi(q, p) .
\end{aligned}
$$

The functions $\phi(q, p)$ are defined in phase space $\Gamma$ and satisfy the condition

$$
\int d^{4} q d^{4} p \phi^{\dagger}(q, p) \phi(q, p)<\infty .
$$

Equation (6) can be derived from Lagrangian given by [5]:

$$
\mathscr{L}=-\left(D^{\mu} \star \phi\right) \star\left(\phi^{*} \star D_{\mu}\right)+m^{2} \phi^{*} \star \phi,
$$

where $D^{\mu}=p^{\mu}-(i / 2)\left(\partial / \partial q_{\mu}\right)$. The association with Wigner formalism is obtained from

$$
f_{W}(q, p)=\phi(q, p) \star \phi^{*}(q, p)
$$

To show this, we multiply the right-hand side of (5) by $\phi^{*}(q, p)$,

$$
\left(p^{2} \star \phi(q, p)\right) \star \phi^{*}(q, p)=m^{2} \phi(q, p) \star \phi^{*}(q, p),
$$

but since $\phi^{*}(q, p) \star p^{2}=m^{2} \phi^{*}(q, p)$, we also have

$$
\phi(q, p) \star\left(\phi^{*}(q, p) \star p^{2}\right)=m^{2} \phi(q, p) \star \phi^{*}(q, p) .
$$

Subtracting (10) from (11), and using the associativity of starproduct, we get

$$
\left\{p^{2}, f_{W}(q, p)\right\}_{M}=0
$$

where the Moyal-bracket is given by

$$
\{a, b\}_{M}=a \star b-b \star a .
$$

Calculating, we obtain

$$
p_{\mu} \frac{\partial f_{W}(q, p)}{\partial q_{\mu}}=0
$$

a well-known result. Other properties of Wigner function, such as nonpositiveness, can be derived analogous to a nonrelativistic case [3].

If we consider the interaction potential $V$, the following density of Lagrangian should be used:

$$
\begin{aligned}
\mathscr{L}= & \frac{-1}{4} \frac{\partial \psi}{\partial q_{\mu}} \frac{\partial \psi^{\dagger}}{\partial q^{\mu}}+\frac{1}{2} i p^{\mu}\left(\psi^{\dagger} \frac{\partial \psi}{\partial q^{\mu}}-\psi \frac{\partial \psi^{\dagger}}{\partial q^{\mu}}\right) \\
& -\left(p^{\mu} p_{\mu}-m^{2}\right) \psi \psi^{\dagger}+U\left(\psi \psi^{\dagger}\right) .
\end{aligned}
$$


This Lagrangian induces the equation

$$
\frac{-1}{4} \frac{\partial^{2} \psi}{\partial q^{\mu} \partial q_{\mu}}-i p^{\mu} \frac{\partial \psi}{\partial q^{\mu}}+\left(p^{\mu} p_{\mu}-m^{2}\right) \psi-V(\psi)=0,
$$

where $V(\psi)=\partial U\left(\psi \psi^{\dagger}\right) / \partial \psi^{\dagger}$.

Solutions for (16) can be obtained from the Green function method. For this proposal, take the function $G=$ $G\left(q^{\mu}, q^{\prime \mu}, p^{\mu}, p^{\prime \mu}\right)$ in which the following is satisfied:

$$
\begin{aligned}
& \frac{-1}{4} \frac{\partial^{2} G}{\partial q^{\mu} \partial q_{\mu}}-i p^{\mu} \frac{\partial G}{\partial q^{\mu}}+\left(p^{\mu} p_{\mu}-m^{2}\right) G \\
& \quad=\delta\left(q^{\mu}-q^{\prime \mu}\right) \delta\left(p^{\mu}-p^{\prime \mu}\right),
\end{aligned}
$$

where $G$ is the Green function. By superposition principle, solution of (17) is given by

$$
\begin{aligned}
\psi\left(q^{\mu}, p^{\mu}\right) & \\
= & \psi_{0}\left(q^{\mu}, p^{\mu}\right) \\
& \quad+\int d^{4} q^{\prime \mu} d^{4} p^{\prime \mu} G\left(q^{\mu}, q^{\prime \mu}, p^{\mu}, p^{\prime \mu}\right) V(\psi),
\end{aligned}
$$

where $\psi_{0}\left(q^{\mu}, p^{\mu}\right)$ is the solution of free case.

We can find the solution of (18) taking its Fourier transform. Defining $F\left[\partial^{2} G / \partial q^{\mu} \partial q_{\mu}\right]=-k^{2} \widetilde{G}\left(k^{\mu}, \eta^{\mu}\right), F\left[\partial G / \partial q^{\mu}\right]=$ $-i k^{\mu} \widetilde{G}\left(k^{\mu}, \eta^{\mu}\right)$, and $F[G]=\widetilde{G}\left(k^{\mu}, \eta^{\mu}\right)$, the following follows:

$$
\begin{aligned}
& \frac{1}{4} k^{2} \widetilde{G}\left(k^{\mu}, p^{\mu}\right)-p^{\mu} k_{\mu} \widetilde{G}\left(k^{\mu}, p^{\mu}\right) \\
& +\left(p^{\mu} p_{\mu}-m^{2}\right) \widetilde{G}\left(k^{\mu}, p^{\mu}\right)=1,
\end{aligned}
$$

where $F[g]$ stands the Fourier transform of function $g$. In this way, we obtain

$$
\widetilde{G}\left(k^{\mu}, p^{\mu}\right)=\frac{\delta\left(p^{\mu}-p^{\prime \mu}\right)}{(1 / 4) k^{2}-p^{\mu} k_{\mu}+\left(p^{\mu} p_{\mu}-m^{2}\right)} .
$$

Then,

$$
\begin{gathered}
G\left(q^{\mu}, q^{\prime \mu}, p^{\mu}, p^{\prime \mu}\right)=\frac{1}{(2 \pi)^{4}} \int d^{4} k^{\mu} e^{-i k^{\mu} q_{\mu}} \widetilde{G}\left(k^{\mu}, p^{\mu}\right) \\
=\frac{1}{(2 \pi)^{4}} \int d^{4} k^{\mu} \frac{\delta\left(p^{\mu}-p^{\prime \mu}\right) e^{-i k^{\mu} q_{\mu}}}{(1 / 4) k^{2}-p^{\mu} k_{\mu}+\left(p^{\mu} p_{\mu}-m^{2}\right)} .
\end{gathered}
$$

The solution is

$$
\begin{aligned}
& \psi\left(q^{\mu}, p^{\mu}\right) \\
& =\psi_{0}\left(q^{\mu}, p^{\mu}\right) \\
& \quad+\int d^{4} q^{\prime \mu} d^{4} p^{\prime \mu} G\left(q^{\mu}, q^{\prime \mu}, p^{\mu}, p^{\prime \mu}\right) V(\psi) .
\end{aligned}
$$
[13]

Wigner function can be derived from Green function by

$$
\begin{aligned}
& f_{W}\left(q^{\mu}, p^{\mu}\right) \\
& =\lim _{q^{\mu^{\prime}} p^{\mu^{\prime}} \rightarrow q^{\mu} p^{\mu}} \exp i\left(\frac{\partial}{\partial q^{\mu}} \frac{\partial}{\partial p^{\mu^{\prime}}}-\frac{\partial}{\partial q^{\mu^{\prime}}} \frac{\partial}{\partial p^{\mu}}\right) \\
& \cdot G\left(q^{\mu}, q^{\prime \mu}, p^{\mu}, p^{\prime \mu}\right) .
\end{aligned}
$$

Equation (23) provides a method to describe the interaction process and scattering theory with physical interpretation in phase space.

\section{Canonical Quantization of Scalar Field in Phase Space}

In this section we construct the formalism of canonical quantization of Klein-Gordon field in phase space. From this formalism we obtain the propagator written in phase space.

From Lagrangian given in (15) we define the conjugate momenta associated with fields $\phi(q, p)$ and $\phi^{\dagger}(q, p)$ by

$$
\pi=\frac{\partial \mathscr{L}}{\left(\partial_{0} \phi\right)}
$$

and

$$
\pi^{\dagger}=\frac{\partial \mathscr{L}}{\left(\partial_{0} \phi^{\dagger}\right)} .
$$

After some calculations, we have

$$
\pi=\frac{-1}{4} \frac{\partial \phi^{\dagger}}{\partial q^{0}}+\frac{1}{2} i p_{0} \phi^{\dagger}=\frac{-1}{2} i p^{0} \star \phi^{\dagger}
$$

and

$$
\pi^{\dagger}=\frac{1}{4} \frac{\partial \phi}{\partial q^{0}}-\frac{1}{2} i p_{0} \phi=\frac{1}{2} i p^{0} \star \phi .
$$

Following usual procedure of quantization, we impose the commutation relations

$$
\left[\pi\left(q, q_{0} ; p\right), \phi\left(q^{\prime}, q_{0} ; p^{\prime}\right)\right]=i \delta\left(q-q^{\prime}\right) \delta\left(p-p^{\prime}\right),
$$

and

$$
\begin{gathered}
{\left[\pi^{\dagger}\left(q, q_{0} ; p\right), \phi^{\dagger}\left(q^{\prime}, q_{0} ; p^{\prime}\right)\right]} \\
\quad=i \delta\left(q-q^{\prime}\right) \delta\left(p-p^{\prime}\right)
\end{gathered}
$$

and the other commutation relations are nulls.

3.1. Annihilation and Creation Operators. The fields $\phi(q, p)$ and $\phi^{\dagger}(q, p)$ may be expanded as

$$
\begin{aligned}
& \phi(q, p) \\
& \quad=\int \frac{d^{3} k}{\left[(2 \pi)^{3} 2 \omega_{k}\right]^{1 / 2}}\left[a(k, p) e^{-i k q}+b^{\dagger}(k, p) e^{i k q}\right]
\end{aligned}
$$


and

$$
\begin{aligned}
\phi^{\dagger} & (q, p) \\
& =\int \frac{d^{3} k}{\left[(2 \pi)^{3} 2 \omega_{k}\right]^{1 / 2}}\left[b(k, p) e^{-i k q}+a^{\dagger}(k, p) e^{i k q}\right],
\end{aligned}
$$

where $\omega_{k}=\left[((1 / 2) \mathbf{k}-\mathbf{p})^{2}+m^{2}\right]^{1 / 2}$ and the canonical variable related to the position is $K$; i.e., $q \longrightarrow k$.

The functions

$$
f_{k}(q)=\frac{e^{-i k q}}{\left[(2 \pi)^{3} 2 \omega_{k}\right]^{1 / 2}}
$$

form an orthonormal set

$$
\int f_{k}^{*}(q) \widetilde{p^{0} \star} f_{k^{\prime}}(q) d^{3} q=\delta^{3}\left(\mathbf{k}-\mathbf{k}^{\prime}\right)
$$

where $A \widetilde{p^{0} \star B}=A\left(p^{0} \star B\right)-\left(p^{0} \star A\right) B$.

In this way, the fields $\phi(q, p)$ and $\phi^{\dagger}(q, p)$ may be written as

$$
\begin{aligned}
& \phi(q, p)=\int \frac{d^{3} k}{\left[(2 \pi)^{3} 2 \omega_{k}\right]^{1 / 2}}\left[a(k, p) f_{k}(q)\right. \\
& \left.+b^{\dagger}(k, p) f_{k}^{*}(q)\right]
\end{aligned}
$$

and

$$
\begin{aligned}
& \phi^{\dagger}(q, p)=\int \frac{d^{3} k}{\left[(2 \pi)^{3} 2 \omega_{k}\right]^{1 / 2}}\left[b(k, p) f_{k}(q)\right. \\
& \left.+a^{\dagger}(k, p) f_{k}^{*}(q)\right] .
\end{aligned}
$$

Inverting (34) and (35) we obtain

$$
\begin{aligned}
& a(k, p)=\int d^{3} q\left[(2 \pi)^{3} 2 \omega_{k}\right]^{1 / 2} f_{k}^{*} \widetilde{p^{0} \star \phi}(q, p) \\
& b(k, p)=\int d^{3} q\left[(2 \pi)^{3} 2 \omega_{k}\right]^{1 / 2} f_{k}^{*} \widetilde{p^{0} \star \phi^{\dagger}}(q, p) ; \\
& a^{\dagger}(k, p)=\int d^{3} q\left[(2 \pi)^{3} 2 \omega_{k}\right]^{1 / 2} \phi^{\dagger}(q, p) \widetilde{p^{0} \star} f_{k} \\
& b^{\dagger}(k, p)=\int d^{3} q\left[(2 \pi)^{3} 2 \omega_{k}\right]^{1 / 2} \phi(q, p) \widetilde{p^{0} \star} f_{k} .
\end{aligned}
$$

We can show that

$$
\left[a(k, p), a^{\dagger}\left(k^{\prime}, p^{\prime}\right)\right]=(2 \pi)^{3} 2 \omega_{k} \delta\left(\mathbf{k}-\mathbf{k}^{\prime}\right),
$$

and

$$
\left[b(k, p), b^{\dagger}(k, p)\right]=(2 \pi)^{3} 2 \omega_{k} \delta\left(\mathbf{k}-\mathbf{k}^{\prime}\right)
$$

The operators $a(k, p), a^{\dagger}(k, p), b(k, p)$, and $b^{\dagger}(k, p)$ play a crucial role in the particle interpretation of the quantized field theory. First, define the operators

$$
N(k, p)=a^{\dagger}(k, p) a(k, p)
$$

and

$$
M(k, p)=b^{\dagger}(k, p) b(k, p)
$$

It is simple to show that $N(k, p)$ and $N\left(k^{\prime}, p^{\prime}\right)$ commute

$$
\left[N(k, p), N\left(k^{\prime}, p^{\prime}\right)\right]=0 .
$$

In analogous sense,

$$
\left[M(k, p), M\left(k^{\prime}, p^{\prime}\right)\right]=0 .
$$

In this case, the eigenstates of these operators may be used to form a basis. Let us denote the eigenvalue of $N(k, p)$ by $n(k, p)$ and the eigenvalue of $M(k, p)$ by $m(k, p)$; i.e.,

$$
\begin{aligned}
N(k, p)|n(k, p)\rangle & =n(k, p)|n(k, p)\rangle, \\
M(k, p)|m(k, p)\rangle & =m(k, p)|m(k, p)\rangle .
\end{aligned}
$$

And now using the commutation relations $\left[N(k, p), a^{\dagger}(k\right.$, $p)]=a^{\dagger}(k, p),[N(k, p), a(k, p)]=-a(k, p),\left[M(k, p), b^{\dagger}(k\right.$, $p)]=b^{\dagger}(k, p)$, and $[M(k, p), b(k, p)]=-b(k, p)$, we find

$$
\begin{aligned}
& N(k, p) a^{\dagger}(k, p)|n(k, \mathrm{p})\rangle \\
& =(n(k, p)+1) a^{\dagger}(k, p)|n(k, p)\rangle, \\
& N(k, p) a(k, p)|n(k, p)\rangle \\
& =(n(k, p)-1) a(k, p)|n(k, p)\rangle, \\
& M(k, p) b^{\dagger}(k, p)|m(k, p)\rangle \\
& =(m(k, p)+1) b^{\dagger}(k, p)|m(k, p)\rangle, \\
& M(k, p) b(k, p)|m(k, p)\rangle \\
& =(m(k, p)-1) b(k, p)|m(k, p)\rangle \text {. }
\end{aligned}
$$

Equations (44) and (45) tell us that if the state $|k, p\rangle$ has eigenvalue $n(k, p)$, the states $a^{\dagger}(k, p)|n(k, p)\rangle$ and $a(k, p)|n(k, p)\rangle$ are eigenstates of $N(k, p)$ with respective eigenvalues $n(k, p)+$ 1 and $n(k, p)-1$. And analogously, we note that (46) and (47) tell us that if the state $|k, p\rangle$ has eigenvalue $m(k, p)$, the states $b^{\dagger}(k, p)|m(k, p)\rangle$ and $b(k, p)|m(k, p)\rangle$ are eigenstates of $M(k, p)$ with respective eigenvalues $m(k, p)+1$ and $m(k, p)-$ 1. So, the operators $a^{\dagger}(k, p)$ and $a(k, p)$ are interpreted as creation and annihilation operators of particles, respectively. Then, analogously, $b^{\dagger}(k, p)$ and $b(k, p)$ can be interpreted as creation and annihilation operators of antiparticles, respectively.

Using the creation and annihilation operators, the Hamiltonian of scalar fields in phase space can be written by

$$
\begin{aligned}
H & =\int \frac{d^{3} k}{(2 \pi)^{3} 2 \omega_{k}}\left[a^{\dagger}(k, p) a(k, p)\right. \\
& \left.+b^{\dagger}(k, p) b(k, p)\right] .
\end{aligned}
$$


We can also show that the particles that are the quantum of the Klein-Gordon field obey the Bose-Einstein statistics. For this, note that

$$
\begin{aligned}
& |n(k, p), m(k, p)\rangle \\
& \quad=\frac{1}{(n(k, p) ! m(k, p) !)^{1 / 2}}\left[a^{\dagger}(k, p)\right]^{n}\left[b^{\dagger}(k, p)\right]^{m} \\
& \quad \cdot|0\rangle .
\end{aligned}
$$

The connection between the solution of free KleinGordon equation, $\varphi(q, p)=\xi\left(p^{\mu}\right) e^{-i k_{\mu} q^{\mu}}$, and the canonical quantization formalism is given by

$$
\varphi(q, p)=\langle 0|\phi(q, p)| k, p\rangle
$$

In sequence, we establish the association between Green function given in (21) and the expression $\langle 0| T[\phi(q$, $\left.p) \phi^{*}\left(q^{\prime}, p^{\prime}\right)\right]|0\rangle$. For this purpose, we consider the Green function

$$
\begin{aligned}
& G\left(q^{\mu}, p^{\mu}, p^{\prime \mu}\right)=\frac{1}{(2 \pi)^{4}} \\
& \quad \cdot \int d^{4} k^{\mu} \frac{\delta\left(p^{\mu}-p^{\prime \mu}\right) e^{-i k^{\mu} q_{\mu}}}{\left((1 / 2) k_{0}-p_{0}\right)^{2}-\left[((1 / 2) \mathbf{k}-\mathbf{p})^{2}+m^{2}\right]},
\end{aligned}
$$

in which it can be written in the form

$$
\begin{aligned}
& G\left(q^{\mu}, p^{\mu}, p^{\prime \mu}\right)=\delta\left(p-p^{\prime}\right) \\
& \quad \cdot \int \frac{d^{3} \mathbf{k} d k_{0}}{(2 \pi)^{4}} \frac{e^{-i k q}}{2 \omega_{k}}\left(\frac{1}{(1 / 2) k_{0}-p_{0}-\omega_{k}+i \epsilon}\right. \\
& \left.\quad-\frac{1}{(1 / 2) k_{0}-p_{0}+\omega_{k}-i \epsilon}\right),
\end{aligned}
$$

where $\omega_{k}^{2}=((1 / 2) \mathbf{k}-\mathbf{p})^{2}$. Using Cauchy Theorem we have

$$
\begin{aligned}
& G\left(q^{\mu}, q^{\prime \mu}, p^{\mu}, p^{\prime \mu}\right)=-2 i \frac{\delta\left(p^{\mu}-p^{\prime \mu}\right)}{(2 \pi)^{3}} \\
& \cdot \int \frac{d^{3} \mathbf{k}}{2 \omega_{k}}\left[\theta\left(q_{0}-q_{0}^{\prime}\right) e^{-i k\left(q_{0}-q_{0}^{\prime}\right)}\right. \\
& \left.+\theta\left(q_{0}^{\prime}-q_{0}\right) e^{i k\left(q_{0}-q_{0}^{\prime}\right)}\right] .
\end{aligned}
$$

In order, substituting (34) and (35) in $\langle 0| T\left[\phi(q, p) \phi^{*}\left(q^{\prime}\right.\right.$, $\left.\left.p^{\prime}\right)\right]|0\rangle$, we obtain

$$
\begin{aligned}
\langle 0| & T\left[\phi(q, p) \phi^{*}\left(q^{\prime}, p^{\prime}\right)\right]|0\rangle \\
= & \int \frac{d^{3} \mathbf{k} d^{3} \mathbf{k}^{\prime}}{(2 \pi)^{6}\left(2 \omega_{k^{\prime}} \omega_{k}\right)^{1 / 2}}\left[\theta\left(q_{0}-q_{0}^{\prime}\right) e^{-i\left(k q-k^{\prime} q^{\prime}\right)}\langle 0|\right. \\
& \cdot a(k, p) a^{\dagger}\left(k^{\prime}, p^{\prime}\right)|0\rangle+\theta\left(q_{0}^{\prime}-q_{o}\right) e^{-i\left(k q^{\prime}-k^{\prime} q\right)}\langle 0| \\
& \left.\cdot a(k, p) a^{\dagger}\left(k^{\prime}, p^{\prime}\right)|0\rangle\right],
\end{aligned}
$$

where the Heaviside function $\theta(x)$ is defined as $\theta(x)=1$ for $x>1$ and $\theta(x)=0$ for $x<1$. Then, using (37) and (38) we obtain

$$
\begin{aligned}
& \left\langle 0\left|T\left[\phi(q, p) \phi^{*}\left(q^{\prime}, p^{\prime}\right)\right]\right| 0\right\rangle=\frac{\delta\left(p^{\mu}-p^{\prime \mu}\right)}{(2 \pi)^{3}} \\
& \cdot \int \frac{d^{3} \mathbf{k}}{2 \omega_{k}}\left[\theta\left(q_{0}-q_{0}^{\prime}\right) e^{-i k\left(q_{0}-q_{0}^{\prime}\right)}\right. \\
& \left.+\theta\left(q_{0}^{\prime}-q_{0}\right) e^{i k\left(q_{0}-q_{0}^{\prime}\right)}\right] .
\end{aligned}
$$

We then have

$$
\begin{gathered}
\left\langle 0\left|T\left[\phi(q, p) \phi^{*}\left(q^{\prime}, p^{\prime}\right)\right]\right| 0\right\rangle \\
=\frac{i}{2} G\left(q^{\mu}, q^{\prime \mu}, p^{\mu}, p^{\prime \mu}\right) .
\end{gathered}
$$

Equation (56) shows us the connection between the propagator $\left\langle 0\left|T\left[\phi(q, p) \phi^{*}\left(q^{\prime}, p^{\prime}\right)\right]\right| 0\right\rangle$ and Green function in phase space.

\section{Thermo Field Dynamics (TFD)}

In this section a brief introduction to TFD formalism is presented [14-18]. In this formalism the thermal average of an observable is given by the vacuum expectation value in an extended Fock space, i.e., $\langle A\rangle=\langle 0(\beta)|A| 0(\beta)\rangle$, where $|0(\beta)\rangle$ is the thermal vacuum. The foundation of the TFD consists in two ingredients: (i) doubling of the original Hilbert space, i.e., the original Hilbert space $\delta$ of the system is doubled leading to an expanded space $\mathcal{S}_{T}=\mathcal{S} \otimes \widetilde{\mathcal{S}}$ and (ii) Bogoliubov transformations. This doubling is defined by a mapping $(\sim)$ : $\mathcal{S} \longrightarrow \widetilde{\mathcal{S}}$ associating each operator say $a$, in $\mathcal{S}$ to two operators in $\mathcal{S}_{T}$, such as

$$
\begin{aligned}
& A=a \otimes 1, \\
& \widetilde{A}=1 \otimes a .
\end{aligned}
$$

The standard doublet notation for an arbitrary bosonic operator $\mathscr{X}$ is

$$
\mathscr{X}^{a}=\left(\begin{array}{c}
\mathscr{X}^{1} \\
\mathscr{X}^{2}
\end{array}\right)=\left(\begin{array}{c}
\mathscr{X} \\
-\widetilde{X}^{\dagger}
\end{array}\right)
$$

where $a, b=1,2$. The physical variables are described by nontilde operators.

The Bogoliubov transformation introduces a rotation in the tilde and nontilde variables. Then the thermal effects are introduced by a Bogoliubov transformation, $\mathscr{U}(\alpha)$, that is defined as

$$
\mathcal{U}(\alpha)=\left(\begin{array}{cc}
u(\alpha) & -v(\alpha) \\
-v(\alpha) & u(\alpha)
\end{array}\right),
$$

where $u^{2}(\alpha)-v^{2}(\alpha)=1$. These quantities $u(\alpha)$ and $v(\alpha)$ are related to the Bose distribution. The parameter $\alpha$ is associated 
with temperature, but, in general, it may be associated with other physical quantities. The Bogoliubov transformations for bosons and fermions are different. For bosons they are given as

$$
\begin{aligned}
& a(k)=c_{B}(\omega) a(k, \beta)+d_{B}(\omega) \tilde{a}^{\dagger}(k, \beta), \\
& a^{\dagger}(k)=c_{B}(\omega) a^{\dagger}(k, \beta)+d_{B}(\omega) \tilde{a}(k, \beta), \\
& \tilde{a}(k)=c_{B}(\omega) \tilde{a}(k, \beta)+d_{B}(\omega) a^{\dagger}(k, \beta), \\
& \tilde{a}^{\dagger}(k)=c_{B}(\omega) \tilde{a}^{\dagger}(k, \beta)+d_{B}(\omega) a(k, \beta),
\end{aligned}
$$

where $\left(a^{\dagger}, \tilde{a}^{\dagger}\right)$ are creation operators and $(a, \widetilde{a})$ are destruction operators, with

$$
\begin{aligned}
& c_{B}^{2}(\omega)=1+f_{B}(\omega), \\
& d_{B}^{2}(\omega)=f_{B}(\omega), \\
& f_{B}(\omega)=\frac{1}{e^{\beta \omega}-1},
\end{aligned}
$$

with $\omega=\omega(k)$.

For fermions the Bogoliubov transformations are

$$
\begin{aligned}
& a(k)=c_{F}(\omega) a(k, \beta)+d_{F}(\omega) \tilde{a}^{\dagger}(k, \beta), \\
& a^{\dagger}(k)=c_{F}(\omega) a^{\dagger}(k, \beta)+d_{F}(\omega) \tilde{a}(k, \beta), \\
& \tilde{a}(k)=c_{F}(\omega) \tilde{a}(k, \beta)-d_{F}(\omega) a^{\dagger}(k, \beta), \\
& \tilde{a}^{\dagger}(k)=c_{F}(\omega) \tilde{a}^{\dagger}(k, \beta)-d_{F}(\omega) a(k, \beta),
\end{aligned}
$$

with

$$
\begin{aligned}
& c_{F}^{2}(\omega)=1-f_{F}(\omega), \\
& d_{F}^{2}(\omega)=f_{F}(\omega), \\
& f_{F}(\omega)=\frac{1}{e^{\beta \omega}+1} .
\end{aligned}
$$

Let us consider a free scalar field in Minkowski space with $\operatorname{diag}\left(g^{\mu \nu}\right)=(+1,-1,-1,-1)$ and then analyze its propagator. Using the Bogoliubov transformation the $\alpha$-dependent scalar field is given by

$$
\phi(x ; \alpha)=\mathscr{U}(\alpha) \phi(x) \mathcal{U}^{-1}(\alpha) .
$$

There is a similar equation for tilde field. The propagator for the scalar field, $\alpha$-dependent, is

$$
\begin{aligned}
G_{0}^{(a b)} & \left(x-x^{\prime} ; \alpha\right) \\
\quad= & i\left\langle 0, \widetilde{0}\left|\tau\left[\phi^{a}(x ; \alpha) \phi^{b}\left(x^{\prime} ; \alpha\right)\right]\right| 0, \widetilde{0}\right\rangle,
\end{aligned}
$$

where $\tau$ is the time ordering operator. Using $|0(\alpha)\rangle=$ $\mathcal{U}(\alpha)|0, \widetilde{0}\rangle$

$$
\begin{aligned}
G_{0}^{(a b)}\left(x-x^{\prime} ; \alpha\right) & =i\left\langle 0(\alpha)\left|\tau\left[\phi^{a}(x) \phi^{b}\left(x^{\prime}\right)\right]\right| 0(\alpha)\right\rangle, \\
& =i \int \frac{d^{4} k}{(2 \pi)^{4}} e^{-i k\left(x-x^{\prime}\right)} G_{0}^{(a b)}(k ; \alpha),
\end{aligned}
$$

where

$$
G_{0}^{(a b)}(k ; \alpha)=\mathcal{U}^{-1}(k ; \alpha) G_{0}^{(a b)}(k) \mathcal{U}(k ; \alpha),
$$

with

$$
\begin{aligned}
& \mathcal{U}(k ; \alpha)=\left(\begin{array}{cc}
u(k ; \alpha) & -v(; \alpha) \\
-v(k ; \alpha) & u(k ; \alpha)
\end{array}\right), \\
& G_{0}^{(a b)}(k)=\left(\begin{array}{cc}
G_{0}(k) & 0 \\
0 & -G_{0}^{*}(k)
\end{array}\right),
\end{aligned}
$$

and

$$
G_{0}(k)=\frac{1}{k^{2}-m^{2}+i \epsilon} .
$$

Then

$$
G_{0}^{(11)}(k ; \alpha)=G_{0}(k)+v^{2}(k ; \alpha)\left[2 \pi i \delta\left(k^{2}-m^{2}\right)\right],
$$

where

$$
\begin{aligned}
v^{2}(k ; \alpha)= & \sum_{s=1}^{d} \sum_{\left\{\sigma_{s}\right\}} 2^{s-1} \sum_{l_{\sigma_{1}}, \ldots, l_{\sigma_{s}}=1}^{\infty}(-\eta)^{s+\sum_{r=1}^{s} l_{\sigma_{r}}} \\
& \cdot \exp \left[-\sum_{j=1}^{s} \alpha_{\sigma_{j}} l_{\sigma_{j}} k^{\sigma_{j}}\right]
\end{aligned}
$$

is the generalized Bogoliubov transformation [19], with $d$ being the number of compactified dimensions, $\eta=1(-1)$ for fermions (bosons), and $\left\{\sigma_{s}\right\}$ denotes the set of all combinations with $s$ elements.

An important note is that in phase space the Green function is dependent on the parameters $q$ and $p$; i.e., $G_{0}^{(a b)}\left(x-x^{\prime} ; \alpha\right)=G_{0}^{(a b)}\left(q^{\mu}-q^{\prime \mu}, p^{\mu}-p^{\prime \mu} ; \alpha\right)$.

\section{Energy-Momentum Tensor for the Scalar Field in Phase Space}

The Lagrangian that describes the scalar field in phase space is given by

$$
\begin{aligned}
\mathscr{L}_{\phi}= & -\frac{1}{4} \frac{\partial \phi}{\partial q_{\mu}} \frac{\partial \phi^{*}}{\partial q^{\mu}}+\frac{1}{2} i p^{\mu}\left(\phi^{*} \frac{\partial \phi}{\partial q^{\mu}}-\phi \frac{\partial \phi^{*}}{\partial q^{\mu}}\right) \\
& -\left(p^{\mu} p_{\mu}-m^{2}\right) \phi \phi^{*} .
\end{aligned}
$$

In order to calculate the Casimir effect, we need the energymomentum tensor that is defined as

$$
\begin{aligned}
& \mathscr{T}^{\mu \nu}=\frac{\partial \mathscr{L}_{\phi}}{\partial\left(\partial_{\mu} \phi\right)} \partial^{\nu} \phi-g^{\mu \nu} \mathscr{L}_{\phi},=-\frac{1}{4}\left(\frac{\partial \phi^{*}}{\partial q_{\mu}} \frac{\partial \phi}{\partial q_{\nu}}\right. \\
&+\left.\frac{\partial \phi}{\partial q_{\mu}} \frac{\partial \phi^{*}}{\partial q_{\nu}}\right)+\frac{1}{2} i p^{\mu}\left(\phi^{*} \frac{\partial \phi}{\partial q_{\nu}}-\phi \frac{\partial \phi^{*}}{\partial q_{\nu}}\right) \\
&-g^{\mu \nu}\left[-\frac{1}{4} \frac{\partial \phi}{\partial q^{\lambda}} \frac{\partial \phi^{*}}{\partial q_{\lambda}}+\frac{1}{2} i p^{\lambda}\left(\phi^{*} \frac{\partial \phi}{\partial q^{\lambda}}-\phi \frac{\partial \phi^{*}}{\partial q^{\lambda}}\right)\right. \\
&\left.-\left(p^{\lambda} p_{\lambda}-m^{2}\right) \phi \phi^{*}\right] .
\end{aligned}
$$


To avoid divergences, the energy-momentum tensor is written at different space-time points as

$$
\begin{aligned}
& \mathscr{T}^{\mu \nu}=\lim _{q^{\prime \mu} \longrightarrow q^{\mu}} \tau\left\{-\frac{1}{4}\left(\frac{\partial \phi^{\prime *}}{\partial q_{\mu}^{\prime}} \frac{\partial \phi}{\partial q_{\nu}}+\frac{\partial \phi}{\partial q_{\mu}} \frac{\partial \phi^{\prime *}}{\partial q_{\nu}^{\prime}}\right)+\frac{1}{2}\right. \\
& \cdot i p^{\mu}\left(\phi^{\prime *} \frac{\partial \phi}{\partial q_{\nu}}-\phi \frac{\partial \phi^{\prime *}}{\partial q_{\nu}^{\prime}}\right)-g^{\mu \nu}\left[-\frac{1}{4} \frac{\partial \phi}{\partial q^{\lambda}} \frac{\partial \phi^{\prime *}}{\partial q_{\lambda}^{\prime}}\right. \\
& +\frac{1}{2} i p^{\lambda}\left(\phi^{\prime *} \frac{\partial \phi}{\partial q^{\lambda}}-\phi \frac{\partial \phi^{\prime *}}{\partial q^{\prime \lambda}}\right) \\
& \left.\left.-\left(p^{\lambda} p_{\lambda}-m^{2}\right) \phi \phi^{\prime *}\right]\right\} \\
& =\lim _{q^{\prime \mu} \longrightarrow q^{\mu}}\left\{\Gamma^{\mu \nu} \tau\left[\phi(q) \phi^{\prime *}\left(q^{\prime}\right)\right]\right\},
\end{aligned}
$$

where $\tau$ is the ordering operator and

$$
\begin{aligned}
\Gamma^{\mu \nu} & =-\frac{1}{4}\left(\frac{\partial}{\partial q_{\mu}^{\prime}} \frac{\partial}{\partial q_{\nu}}+\frac{\partial}{\partial q_{\mu}} \frac{\partial}{\partial q_{\nu}^{\prime}}\right)+\frac{1}{2} i p^{\mu}\left(\frac{\partial}{\partial q_{\nu}}\right. \\
& \left.-\frac{\partial}{\partial q_{\nu}^{\prime}}\right)-g^{\mu \nu}\left[-\frac{1}{4} \frac{\partial}{\partial q^{\lambda}} \frac{\partial}{\partial q_{\lambda}^{\prime}}\right. \\
& \left.+\frac{1}{2} i p^{\lambda}\left(\frac{\partial}{\partial q^{\lambda}}-\frac{\partial}{\partial q^{\prime \lambda}}\right)-\left(p^{\lambda} p_{\lambda}-m^{2}\right)\right] .
\end{aligned}
$$

The vacuum expectation value of the energy-momentum tensor is

$$
\left\langle\mathscr{T}^{\mu \nu}(x)\right\rangle=\lim _{q^{\prime \mu} \longrightarrow q^{\mu}}\left\{\Gamma^{\mu \nu}\left\langle 0\left|\tau\left[\phi(q) \phi^{\prime *}\left(q^{\prime}\right)\right]\right| 0\right\rangle\right\} .
$$

The scalar field propagator in phase space is defined as

$$
\begin{aligned}
& G_{0}\left(q^{\mu}-q^{\prime \mu}, p^{\mu}-p^{\prime \mu}\right)=\left\langle 0\left|\tau\left[\phi(q) \phi^{\prime *}\left(q^{\prime}\right)\right]\right| 0\right\rangle \\
& =\int \frac{d^{4} k}{(2 \pi)^{4}} \frac{\delta\left(p^{\mu}-p^{\prime \mu}\right) e^{i \kappa_{\mu}\left(q^{\mu}-q^{\prime \mu}\right)}}{\left((1 / 4) \kappa^{2}-i \kappa_{\mu}\left(p^{\mu}-p^{\prime \mu}\right)+\left(p^{\mu}-p^{\prime \mu}\right)+m^{2}\right)} .
\end{aligned}
$$

Using the identity

$$
\frac{1}{\chi^{\mu} \chi_{\mu}+M^{2}}=\int_{0}^{\infty} e^{-t\left(\chi^{\mu} \chi_{\mu}-M^{2}\right)} d t
$$

with $M^{2}=m^{2}-2\left(p^{\mu}-p^{\prime \mu}\right)\left(p^{\mu}-p^{\prime \mu}\right)$, the propagator becomes

$$
\begin{aligned}
G_{0}\left(q^{\mu}-q^{\prime \mu}, p^{\mu}-p^{\prime \mu}\right) \\
=\frac{64 M^{2}}{\pi^{2}} \delta\left(p^{\mu}-p^{\prime \mu}\right) e^{-2\left(p^{\mu}-p^{\prime \mu}\right)\left(q^{\mu}-q^{\prime \mu}\right)} \\
\quad \times\left(\frac{M\left|q-q^{\prime}\right|}{2}\right)^{-1} \kappa_{1}\left(\frac{M\left|q-q^{\prime}\right|}{2}\right),
\end{aligned}
$$

where $\kappa_{v}(z)$ is the Bessel function. Using the doublet notation, the physical energy-momentum tensor in terms of the $\alpha$ parameter is

$$
\mathbb{T}^{\mu \nu(a b)}(q)=\lim _{q^{\prime \mu} \longrightarrow q^{\mu}}\left\{\Gamma^{\mu \nu} \bar{G}_{0}^{a b}\left(q^{\mu}-q^{\prime \mu}, p^{\mu}-p^{\prime \mu} ; \alpha\right)\right\},
$$

where $\mathbb{T}^{\mu \nu(a b)}(q ; \alpha)=\left\langle\mathscr{T}^{\mu \nu(a b)}(q ; \alpha)\right\rangle-\left\langle\mathscr{T}^{\mu \nu(a b)}(q)\right\rangle$ and

$$
\begin{aligned}
\bar{G}_{0}^{(a b)} & \left(q^{\mu}-q^{\prime \mu}, p^{\mu}-p^{\prime \mu} ; \alpha\right) \\
= & G_{0}^{(a b)}\left(q^{\mu}-q^{\prime \mu}, p^{\mu}-p^{\prime \mu} ; \alpha\right) \\
& -G_{0}^{(a b)}\left(q^{\mu}-q^{\prime \mu}, p^{\mu}-p^{\prime \mu}\right) .
\end{aligned}
$$

\section{Some Applications}

In this section the Stefan-Boltzmann law and the Casimir effect at finite temperature are calculated.

6.1. Stefan-Boltzmann Law. Here the $\alpha$ parameter is $\alpha=$ $(\beta, 0,0,0)$ and the generalized Bogoliubov transformation takes the form

$$
v^{2}(\beta)=\sum_{l_{0}=1}^{\infty} e^{-\beta k^{0} l_{0}}
$$

and the Green function becomes

$$
\begin{aligned}
\bar{G}_{0}^{(a b)} & \left(q^{\mu}-q^{\prime \mu}, p^{\mu}-p^{\prime \mu} ; \beta\right) \\
\quad= & 2 \sum_{l_{0}=1}^{\infty} G_{0}\left(q^{\mu}-q^{\prime \mu}-i \beta l_{0} n_{0}, p^{\mu}-p^{\prime \mu}\right),
\end{aligned}
$$

where $n_{0}=(1,0,0,0)$ is a time-like vector. For $\mu=\nu=0$, the energy-momentum tensor becomes

$$
\begin{aligned}
& \mathbb{T}^{00(11)}(\beta)=2 \lim _{q^{\prime \mu} \rightarrow q^{\mu}}\left\{\sum _ { l _ { 0 } = 1 } ^ { \infty } \Gamma ^ { 0 0 } G _ { 0 } \left(q^{\mu}-q^{\prime \mu}\right.\right. \\
& \left.\left.-i \beta l_{0} n_{0}, p^{\mu}-p^{\prime \mu}\right)\right\}=\sum_{l_{0}} \frac{128}{\pi^{2}} M^{2} \delta\left(p^{\mu}-p^{\prime \mu}\right) \\
& \cdot e^{2 i\left(p_{0}-p_{0}^{\prime}\right)\left(\beta l_{0}\right)}\left\{\kappa_{1}\left(\frac{M}{2} i l_{0} \beta\right) \times\left(p^{\mu} p_{\mu}+M^{2}\right)\right. \\
& \cdot\left(\frac{M}{2} i l_{0} \beta\right)^{-1}-\frac{1}{8 l_{0}^{2} \beta^{2}}\left[12 \kappa_{0}\left(\frac{M}{2} i l_{0} \beta\right)\right. \\
& \left.\left.+\frac{1}{i M l_{0} \beta}\left(24-i^{2} l_{0}^{2} M^{2} \beta^{2}\right) \kappa_{1}\left(\frac{M}{2} i l_{0} \beta\right)\right]\right\} .
\end{aligned}
$$

This is the Stefan-Boltzmann law in phase space. This result becomes $\mathbb{T}^{00(11)}(\beta) \sim T^{4}$, when it is projected in the usual quantum mechanics space. It is possible to introduce a "temperature", $\varepsilon$, in the momenta space which in fact 
represents a thermal energy. Using the same procedure above, we get

$$
\begin{aligned}
& T^{00(11)}(\beta, p, \varepsilon)=\sum_{j, l_{0}} \frac{128}{\pi^{2}} \mu_{j}^{2} \delta(-i \varepsilon j) \\
& \cdot e^{2(\varepsilon j)\left(\beta l_{0}\right)}\left\{\kappa_{1}\left(\frac{\mu_{j}}{2} i l_{0} \beta\right) \times\left(p^{\mu} p_{\mu}+\mu_{j}^{2}\right)\right. \\
& \cdot\left(\frac{\mu_{j}}{2} i l_{0} \beta\right)^{-1}-\frac{1}{8 l_{0}^{2} \beta^{2}}\left[12 \kappa_{0}\left(\frac{\mu_{j}}{2} i l_{0} \beta\right)\right. \\
& \left.\left.+\frac{1}{i \mu_{j} l_{0} \beta}\left(24-i^{2} l_{0}^{2} \mu_{j}^{2} \beta^{2}\right) \kappa_{1}\left(\frac{\mu_{j}}{2} i l_{0} \beta\right)\right]\right\},
\end{aligned}
$$

where $\mu_{j}^{2}=m^{2}+2(\varepsilon j)^{2}$. It is interesting to note the limit of such an expression when $m \longrightarrow 0$ and $\beta \longrightarrow \infty$; it shows that when the temperature goes to zero the thermal energy follows which means a correlation between them. The symplectic structure of phase space allows one to introduce both finite temperature and energy which is one advantage of our method.

6.2. Casimir Effect at Zero Temperature. Now $\alpha=(0,0,0$, i2d); then

$$
v^{2}(d)=\sum_{l_{3}=1}^{\infty} e^{-i 2 d k^{3} l_{3}}
$$

and thus

$$
\begin{aligned}
\bar{G}_{0} & \left(q^{\mu}-q^{\prime \mu}, p^{\mu}-p^{\prime \mu} ; d\right) \\
& =2 \sum_{l_{3}=1}^{\infty} G_{0}\left(q^{\mu}-q^{\prime \mu}-2 d l_{3} n_{3}, p^{\mu}-p^{\prime \mu}\right)
\end{aligned}
$$

is the Green function with $n_{3}=(0,0,0,1)$, being the space-like vector. The energy-momentum tensor for this case becomes

$$
\begin{aligned}
& \mathbb{T}^{33(11)}(\beta)=2 \lim _{q^{\prime \mu} \longrightarrow q^{\mu}}\left\{\sum _ { l _ { 3 } = 1 } ^ { \infty } \Gamma ^ { 3 3 } G _ { 0 } \left(q^{\mu}-q^{\prime \mu}\right.\right. \\
& \left.\left.-2 d l_{3} n_{3}, p^{\mu}-p^{\prime \mu}\right)\right\}=\sum_{l_{3}} \frac{128}{\pi^{2}} M^{2} \delta\left(p^{\mu}-p^{\prime \mu}\right) \\
& \cdot e^{2 i\left(p_{3}-p_{3}^{\prime}\right) d l_{3}} \times\left\{\kappa_{1}\left(\frac{M}{2} i d l_{3}\right)+\left(-p^{\mu} p_{\mu}+M^{2}\right)\right. \\
& \cdot\left(\frac{M}{2} i d l_{3}\right)^{-1}+\frac{1}{16 l_{3}^{2} d^{2}}\left[6 \kappa_{0}\left(d l_{3} M\right)\right. \\
& \left.\left.+\frac{\left(12+d^{2} l_{3}^{2} M^{2}\right)}{d l_{3} M} \kappa_{1}\left(d l_{3} M\right)\right]\right\} \text {. }
\end{aligned}
$$

It is the Casimir pressure at zero temperature in phase space. In the standard quantum mechanics space the usual result is recovered. The introduction of a thermal energy at this point is similar to finite temperature once they are related to each other as already mentioned. Hence in the next section we analyze the Casimir effect for a finite temperature.

6.3. Casimir Effect at Finite Temperature. In this case the $\alpha$ parameter is chosen as $\alpha=(\beta, 0,0, i 2 d)$. The generalized Bogoliubov transformation is given by

$$
\begin{aligned}
v^{2}(\beta, d)= & \sum_{l_{0}=1}^{\infty} e^{-\beta k^{0} l_{0}}+\sum_{l_{3}=1}^{\infty} e^{-i 2 d k^{3} l_{3}} \\
& +2 \sum_{l_{0}, l_{3}=1}^{\infty} e^{-\beta k^{0} l_{0}-i 2 d k^{3} l_{3}} .
\end{aligned}
$$

The first two terms are associated with the Stefan-Boltzmann law and the Casimir effect at zero temperature. The Green function of the third term in (89), our interest here, is

$$
\begin{aligned}
\bar{G}_{0} & \left(q^{\mu}-q^{\prime \mu}, p^{\mu}-p^{\prime \mu} ; \beta, d\right) \\
\quad=4 & \sum_{l_{0}, l_{3}=1}^{\infty} G_{0}\left(q^{\mu}-q^{\prime \mu}-i \beta l_{0} n_{0}-2 d l_{3} n_{3}, p^{\mu}-p^{\prime \mu}\right) .
\end{aligned}
$$

Then the Casimir pressure at finite temperature in phase space is given as

$$
\begin{aligned}
& \mathbb{T}^{33(11)}(\beta, d)=4 \lim _{q^{\prime \mu} \rightarrow q^{\mu}}\left\{\sum _ { l _ { 0 } , l _ { 3 } = 1 } ^ { \infty } \Gamma ^ { 3 3 } G _ { 0 } \left(q^{\mu}-q^{\prime \mu}-i \beta l_{0} n_{0}\right.\right. \\
& \left.\left.-2 d l_{3} n_{3}, p^{\mu}-p^{\prime \mu}\right)\right\}=\sum_{l_{0}, l_{3}} \frac{256 M^{2}}{\pi^{2}} \delta\left(p^{\mu}-p^{\prime \mu}\right) \\
& \cdot e^{2 i\left(p_{3}-p_{3}^{\prime}\right) d l_{3}} e^{2 i\left(p_{0}-p_{0}^{\prime}\right)\left(\beta l_{0}\right)} \times\left\{\kappa_{1}\left[\frac{M}{2} \sqrt{\left(2 d l_{3}\right)^{2}+\left(l_{0} \beta\right)^{2}}\right]\right. \\
& +\left(-p^{\mu} p_{\mu}+M^{2}\right)\left[\frac{M}{2} \sqrt{\left(2 d l_{3}\right)^{2}+\left(l_{0} \beta\right)^{2}}\right]^{-1} \\
& +\frac{1}{2}\left[\frac{\left(3\left(2 d l_{3}\right)^{2}-\left(l_{0} \beta\right)^{2}\right)}{\left(\left(2 d l_{3}\right)^{2}+\left(l_{0} \beta\right)^{2}\right)^{2}} \kappa_{0}\left(\frac{M}{2} \sqrt{\left(2 d l_{3}\right)^{2}+\left(l_{0} \beta\right)^{2}}\right)\right. \\
& +\frac{\left(48\left(2 d l_{3}\right)^{2}+\left(2 d l_{3}\right)^{4} M^{2}-\left(l_{0} \beta\right)^{2}\left(16+\left(l_{0} M \beta\right)^{2}\right)\right)}{M\left(\left(2 d l_{3}\right)^{2}+\left(l_{0} \beta\right)^{2}\right)^{5 / 2}} \\
& \left.\left.\cdot \kappa_{1}\left(\frac{M}{2} \sqrt{\left(2 d l_{3}\right)^{2}+\left(l_{0} \beta\right)^{2}}\right)\right]\right\} \text {. }
\end{aligned}
$$

This result contains the effect of both time and space compactification. In the same way, in the usual quantum mechanics space the standard result is recovered. 


\section{Conclusions}

In this article the introduction of temperature in phase space was explored. We defined the scalar field in phase space by means of using invariants of the respective relativistic algebra. Then we presented the energy-momentum tensor in such a space. This result was used to implement the prescription of Thermo Field Dynamics, which allows dealing with some phenomena at finite temperature, such as the analogous Casimir effect and Stefan-Boltzmann law. We point out that we projected the mean energy and pressure in the space of coordinates in order to recover the results of literature. If we project our result in the momentum space we should obtain a fundamental energy associated with the given temperature. Such a result should be better understood since the existence of this thermal energy affects the interpretation of phase space.

\section{Data Availability}

No data were used to support this study.

\section{Conflicts of Interest}

The authors declare that they have no conflicts of interest.

\section{Acknowledgments}

The work by A. F. Santos is supported by CNPq Project 308611/2017-9.

\section{References}

[1] E. Wigner, "On the quantum correction for thermodynamic equilibrium," Physical Review A: Atomic, Molecular and Optical Physics, vol. 40, no. 5, pp. 749-759, 1932.

[2] M. Hillery, R. F. O'Connell, M. O. Scully, and E. P. Wigner, "Distribution functions in physics: fundamentals," Physics Reports, vol. 106, no. 3, pp. 121-167, 1984.

[3] M. D. Oliveira, M. C. Fernandes, F. C. Khanna, A. E. Santana, and J. D. Vianna, "Symplectic quantum mechanics," Annals of Physics, vol. 312, no. 2, pp. 492-510, 2004.

[4] R. G. Amorim, M. C. Fernandes, F. C. Khanna, A. E. Santana, and J. D. Vianna, "Non-commutative geometry and symplectic field theory," Physics Letters A, vol. 361, no. 6, pp. 464-471, 2007.

[5] R. G. G. Amorim, F. C. Khanna, A. P. C. Malbouisson, J. M. C. Malbouisson, and A. E. Santana, "Realization of the noncommutative Seiberg-Witten gauge theory by fields in phase space," International Journal of Modern Physics A, vol. 30, no. 22, 2015.

[6] T. Matsubara, "A new approach to quantum-statistical mechanics," Progress of Theoretical and Experimental Physics, vol. 14, no. 4, pp. 351-378, 1955.

[7] F. C. Khanna, A. P. Malbouisson, J. M. Malbouisson, and A. E. Santana, Themal Quantum Field Theory: Algebraic Aspects And Applications, World Scientific Publishing Co. Pte. Ltd., Hackensack, NJ, USA, 2009.

[8] H. B. G. Casimir, "On the attraction between two perfectly conducting plates," Proceedings of the Koninklijke Nederlandse Academie van Wetenschappen. Series B: Physical sciences, vol. 51, pp. 793-795, 1948.
[9] S. C. Ulhoa, A. F. Santos, and F. C. Khanna, "Galilean covariance, Casimir effect and Stefan-Boltzmann law at finite temperature," International Journal of Modern Physics A, vol. 32, no. 16, 2017.

[10] M. J. Sparnaay, "Measurements of attractive forces between flat plates," Physica A: Statistical Mechanics and Its Applications, vol. 24, no. 6-10, pp. 751-764, 1958.

[11] S. K. Lamoreaux, "Demonstration of the casimir force in the 0.6 to $6 \mu \mathrm{m}$ range," Physical Review Letters, vol. 78, no. 5, 1997.

[12] U. Mohideen and A. Roy, "Precision measurement of the Casimir force from 0.1 to $0.9 \mu \mathrm{m}$," Physical Review Letters, vol. 81, p. 4549, 1998.

[13] R. G. Amorim, F. C. Khanna, A. E. Santana, and J. D. Vianna, "Perturbative symplectic field theory and Wigner function," Physica A: Statistical Mechanics and its Applications, vol. 388, no. 18, pp. 3771-3778, 2009.

[14] Y. Takahashi and H. Umezawa, "Thermo field dynamics," Collective Phenomena, vol. 2, pp. 55-80, 1975, (Reprinted in International Journal of Modern Physics B, vol. 10, no. 1755, 1996).

[15] Y. Takahashi, H. Umezawa, and H. Matsumoto, Thermofield Dynamics and Condensed States, North-Holland, Amsterdam, 1982.

[16] H. Umezawa, Advanced Field Theory: Micro, Macro and Thermal Physics, AIP, New York, NY, USA, 1993.

[17] A. E. Santana and F. C. Khanna, "Lie groups and thermal field theory," Physics Letters A, vol. 203, no. 2-3, pp. 68-72, 1995.

[18] A. E. Santana, F. C. Khanna, H. Chu, and Y. Chang, "Thermal Lie groups, classical mechanics, and thermofield dynamics," Annals of Physics, vol. 249, no. 2, pp. 481-498, 1996.

[19] F. C. Khanna, A. P. Malbouisson, J. M. Malbouisson, and A. E. Santana, "Quantum fields in toroidal topology" Annals of Physics, vol. 326, no. 10, pp. 2634-2657, 2011. 

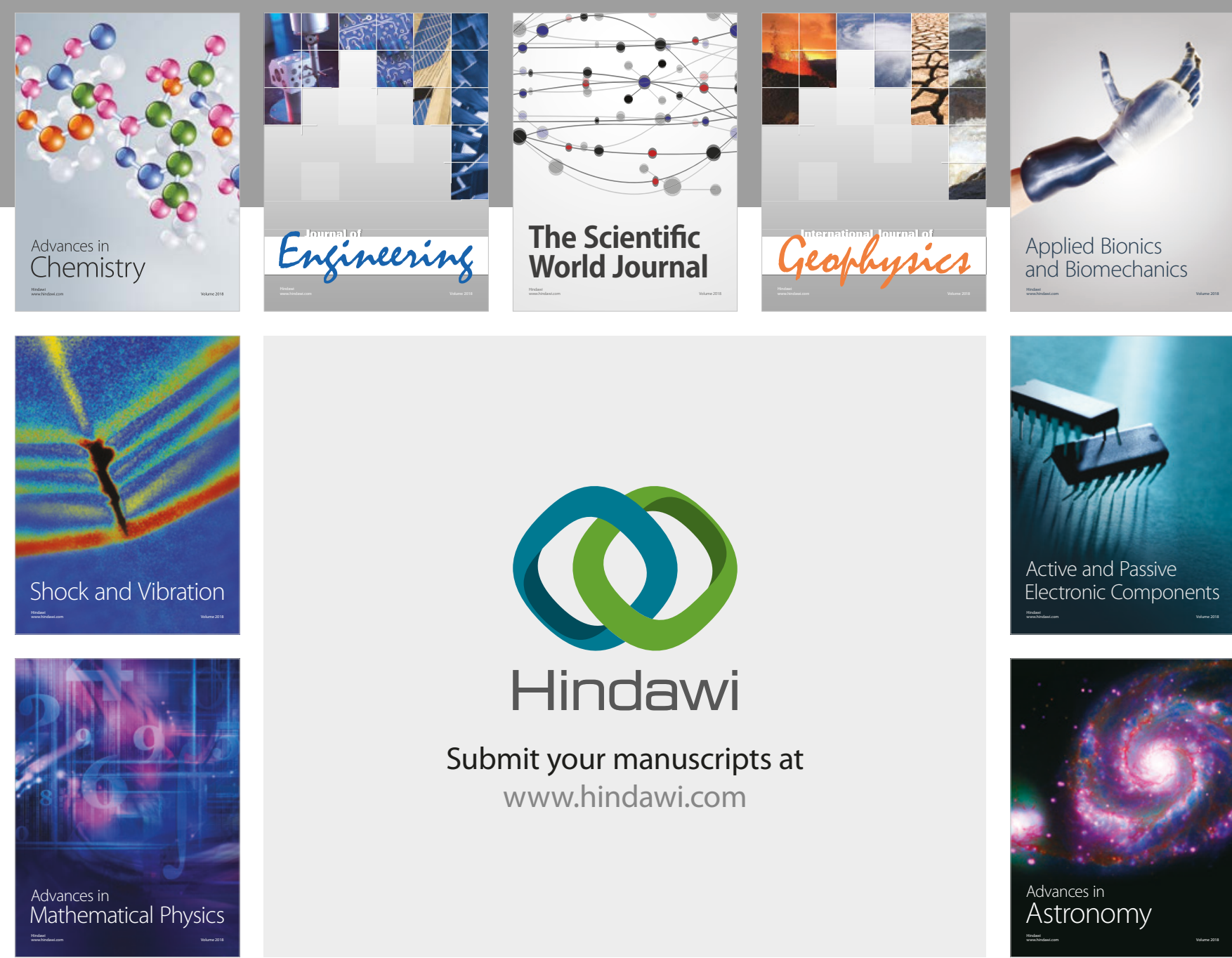

Submit your manuscripts at

www.hindawi.com

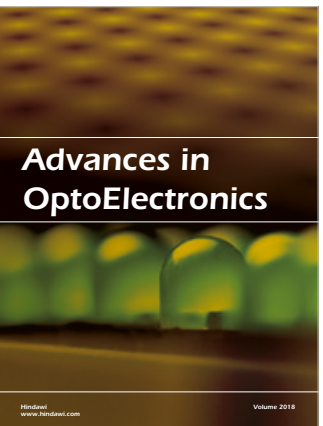

\section{Rotcting Machinery}
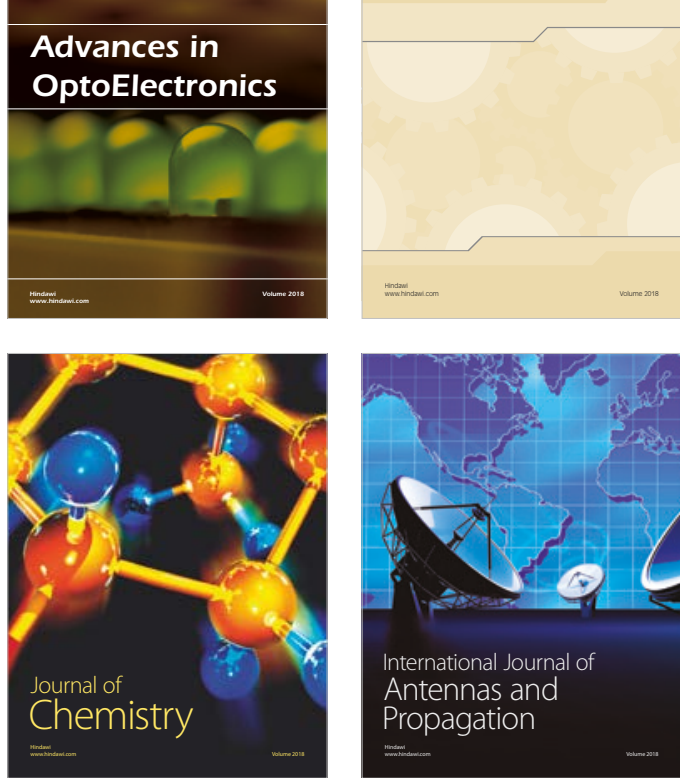

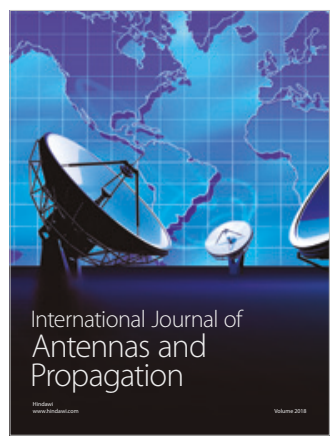

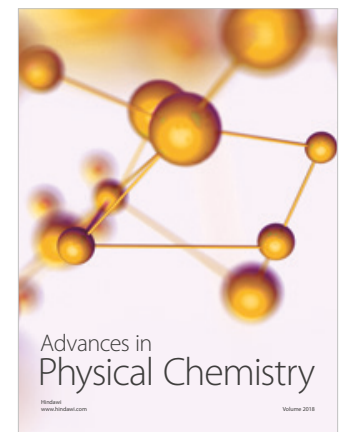

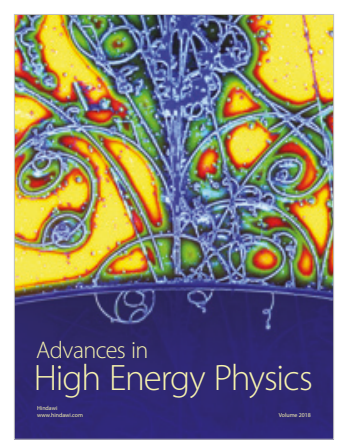

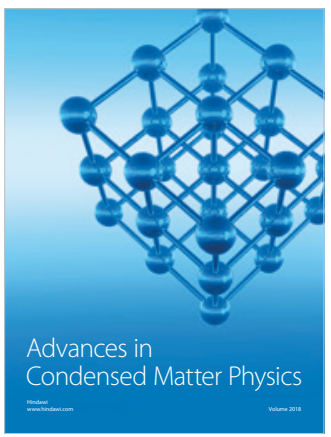

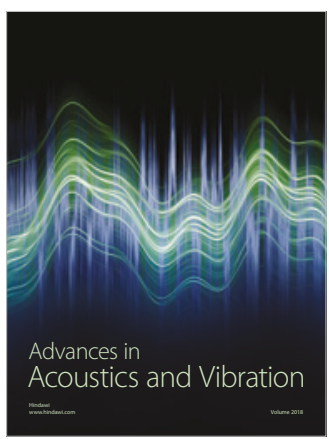

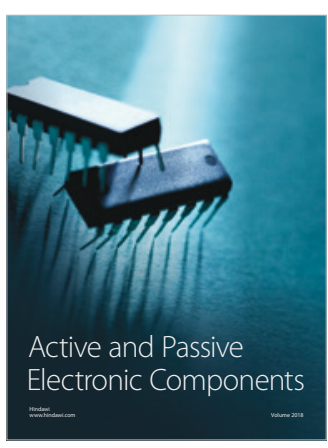
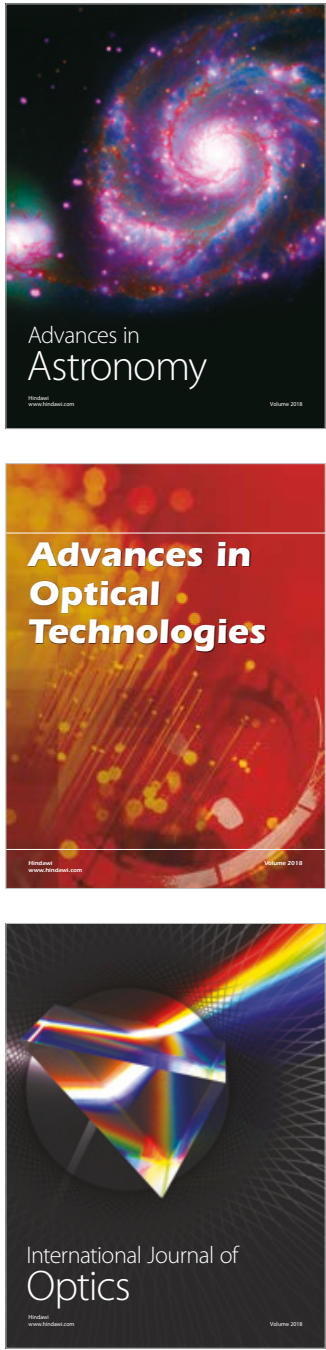